\title{
Tunnel Monitoring Method Based on Wireless Sensor Network
}

\author{
Kiwamu TSUNO \\ Ryo HIRATA \\ Tunnel Engineering Laboratory, Structures Technology Division
}

\begin{abstract}
A monitoring method using a wireless sensor network was developed to lighten the data acquisition workload and reduce the costs for monitoring railway tunnels. A relay method was proposed for transmitting the data over a long distance. The performance of the monitoring system was verified by means of field tests in actual railway tunnels. This paper also describes a data processing method which eliminates the short-term change caused by daily temperature changes in order to utilize the real-time monitoring data for tunnel maintenance. It is found that a low-pass filter is an effective tool for eliminating the short-term change.
\end{abstract}

Keywords: tunnel, monitoring, wireless sensor network, data processing

\section{Introduction}

Long-term monitoring of distorted railway tunnels, such as that of inner space displacement, is normally carried out based on manual measurement. As the measurement work has to be carried out within limited timeframes, mostly in the middle of the night, it is difficult to obtain measurement data at short intervals. On the other hand, automatic monitoring methods with sophisticated devices such as light wave distance measuring instruments and laser range finder, which carry out real-time data acquisition, necessitate a huge amount of work and expense for installing and maintaining cables as well as costly equipment, especially in the tunnels which are usually long in the longitudinal direction. The authors therefore prepare com pact and light wireless sensors and investigate the reduction effect on the data acquisition workload and costs for the long-term monitoring of railway tunnels via wireless data transmission $[1,2]$.

If automatic monitoring is carried out using wireless sensors, a lot of accumulated data are affected by shortterm change caused by daily temperature changes. The daily temperature changes, which are not large inside tunnels as compared with bridges and viaducts, may affect the monitoring data near tunnel entrances, which are likely to be distorted by unsymmetrical earth pressure and landslides. A data processing method which eliminates the short-term change caused by daily temperature changes is therefore developed in order to efficiently utilize the realtime monitoring data for tunnel maintenance.

\section{Tunnel monitoring method based on wireless sen- sor network}

\subsection{Wireless sensor}

Figure 1 shows the outline of the tunnel monitoring system with wireless sensor network proposed in this research. Sensors such as crack width gauges are fixed on tunnel linings and connected with end devices. Measurement data are transmitted from the end devices to a coordinator connected with a computer, which is used as a data logger. When the coordinator is installed at a tun-

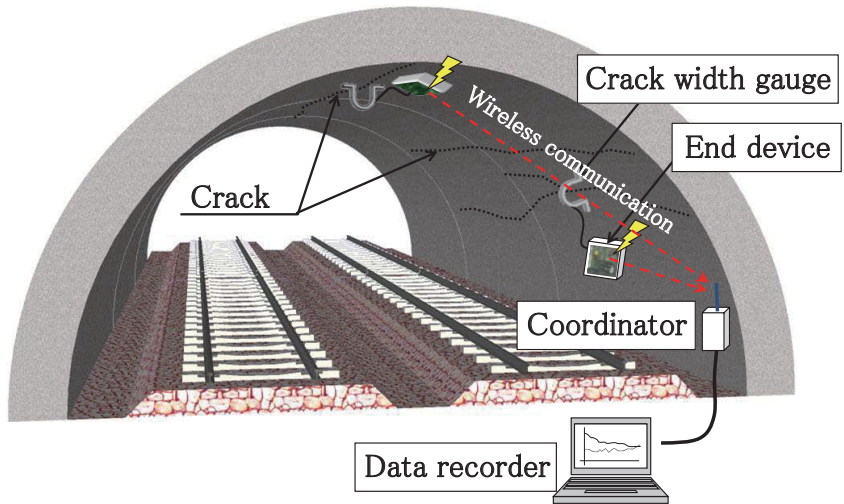

Fig. 1 Outline of the tunnel monitoring system

nel entrance, it is possible to transmit measurement data without cables for data transmission inside the tunnel. As it would not be reasonable to prepare power-generating facilities for end devices as well as installing cables for power feeding, it is required to save the consumed power of end devices for the data transmission. A wireless application based on ZigBee is therefore used in the proposed monitoring system to save the consumed power although they are inferior as compared with a Wi-Fi network in transmission distance and data signal rate. A small signal conditioner with power saving has been developed for strain-type sensors such as $\pi$-type crack width gauges used in the system. The development of a signal conditioner and the use of the sleep mode enable the end devices to run for a long time with AA lithium primary batteries.

In the case of monitoring inside tunnels, end devices need to be made lightweight and thin because all devices have to be installed within limited space and are desired to have good usability. The end devices, including temperature sensors, amplifiers and batteries, are put in waterproof containers, which are $10 \mathrm{~cm}$ in width, $10 \mathrm{~cm}$ in depth and $3.5 \mathrm{~cm}$ in height, as shown in Fig. 2. The developed wireless sensors can transmit data every 1 to 10 minutes and are compatible with some of displacement meters, water-level gauges and axial force meters for rock bolts as well as the $\pi$-type crack width gauges. A performance test carried out in a defunct tunnel shows that the maximum transmission distance inside tunnels is $140 \mathrm{~m}$ [1]. 


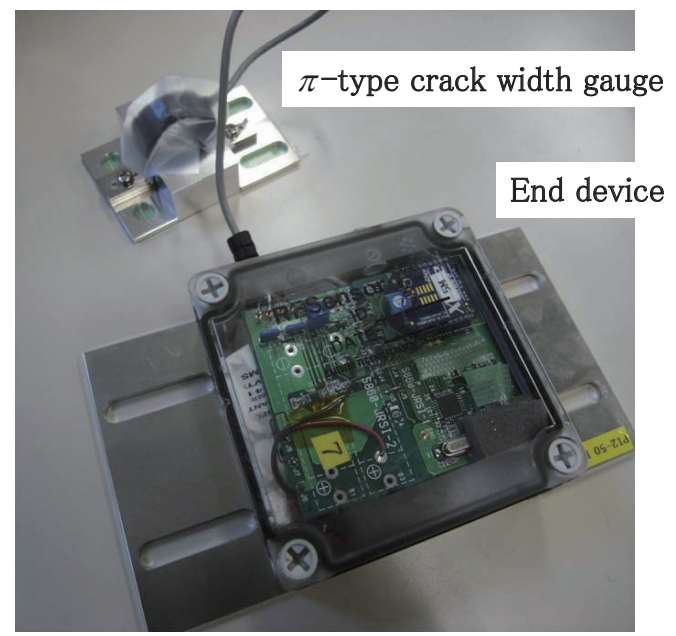

Fig. 2 Wireless sensor (end device)

\subsection{Field test}

To verify the applicability of the proposed system, which would use a wireless sensor network to carry out on-site monitoring, a field test is performed at Tunnel A, which is a single-track railway tunnel in an unelectrified section $[3,4]$. End devices and $\pi$-type crack width gauges are fixed on tunnel linings at three locations, namely near the tunnel entrance, and 15 and $87 \mathrm{~m}$ from the tunnel entrance, as shown in Fig. 3. Measurement data for crack width, temperature and battery voltage are transmitted to a coordinator positioned on the side wall near the tunnel entrance every 8.5 minutes. Four AA lithium primary batteries are placed in the end devices. The measurement data are recorded in a computer which is installed in a shed outside the tunnel and connected with the coordinator via an USB cable.

Figure 4 shows the change in battery voltages. It is found that the battery voltages have been stable for more than one year and suddenly reduce 15 to 18 months after the start of monitoring. The reduction in the battery voltage causes data acquisition to stop. This halting of data acquisition is observed a little earlier at the measurement location near the tunnel entrance, where the change of temperature is large, than at the other locations.

The measurement data for crack width and temperature are shown in Fig.5, which shows that the data is ac-

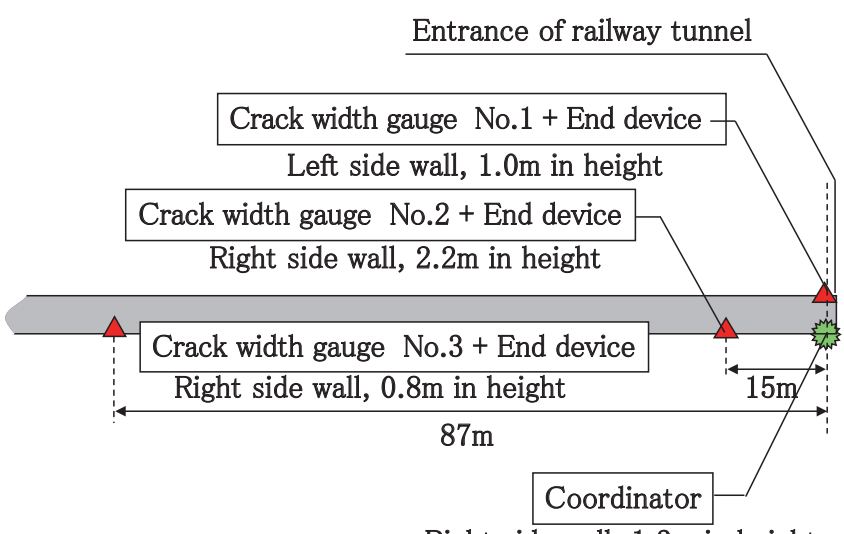

Right side wall, $1.3 \mathrm{~m}$ in height

Fig. 3 Outline of the field test [3]

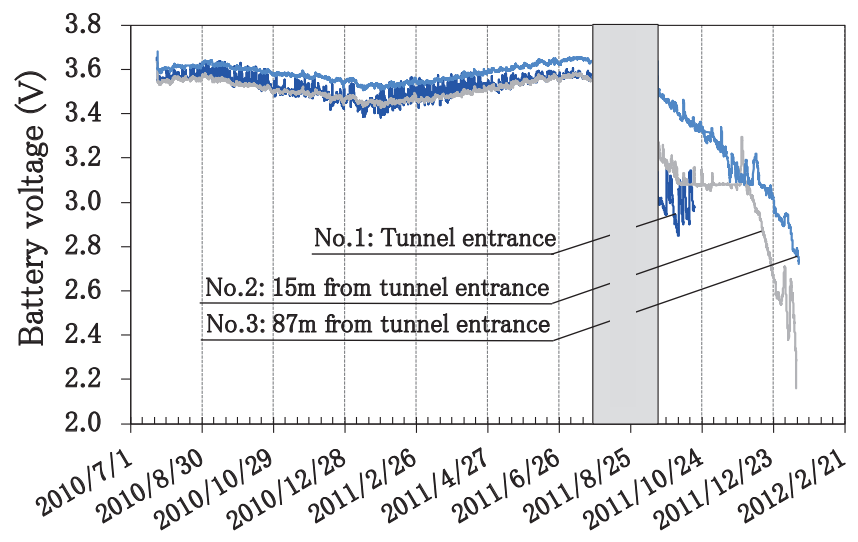

Fig. 4 Change of battery voltage

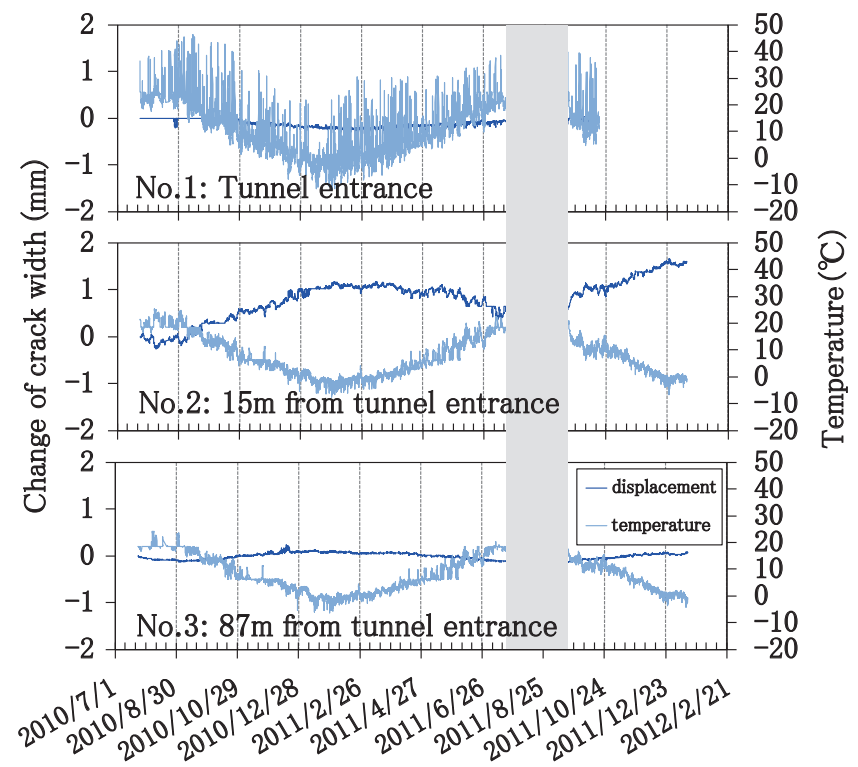

Fig. 5 Change of crack width and temperature

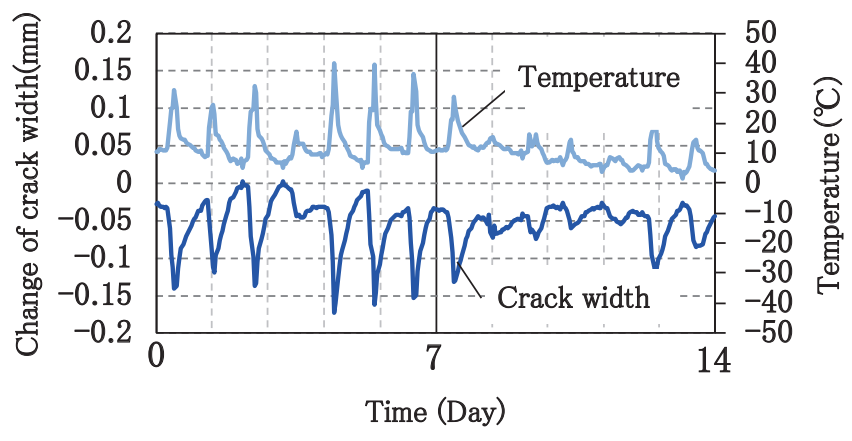

Fig. 6 Measurement data at the tunnel entrance (No.1)

quired without incident. Figure 6 shows the measurement data gathered near the tunnel entrance for 14 days. It is found that the crack width values change with daily temperature changes and become smaller as the temperature becomes higher. As mentioned above, it is verified that it is possible to acquire the measurement data for crack width and temperature just before the reduction in battery voltage causes data acquisition to stop. 


\subsection{Long-distance data transmission with relay sen- sors}

As railway tunnels are usually long in the longitudinal direction, the required transmission distance tends to be long. On the other hand, the transmission distance from end devices to a coordinator is limited. This research therefore investigates a method of long-distance transmission via relay sensors.

Wireless application of ZigBee can construct a network as shown in Fig. 7 (a), in which end devices also function as relay sensors. To keep this kind of network, it is necessary to leave the power supply of end devices switched on and, consequently, exchange the batteries quite frequently. In addition, it is necessary to put a huge amount of end devices on tunnel linings even at the sections not situated at measurement locations. This research therefore proposes a data transmission method for use in railway tunnels with sensors specific for relay as shown in Fig. 7 (b). The relay sensors are also placed in waterproof containers, which measure $10 \mathrm{~cm}$ in width, $10 \mathrm{~cm}$ in depth and $3.5 \mathrm{~cm}$ in height. Although the relay sensors are specifically designed for the relay of data transmission, they also send the temperature data obtained by a built-in thermometer and battery voltage.

To verify the performance of relay sensors, a field test is carried out at Tunnel $\mathrm{B}$, which is a single-track railway tunnel in an unelectrified section as shown in Fig. 8 [5]. Five relay sensors are positioned on the tunnel side wall at a height of $1.6 \mathrm{~m}$ from F.L. as shown in Fig. 9 (b), while a coordinator connected with a computer via a USB cable is installed $137 \mathrm{~m}$ away from the tunnel entrance. The distance from relay sensor 5 to the coordinator is $442 \mathrm{~m}$. Four sets of $\pi$-type crack width gauges and end devices are fixed

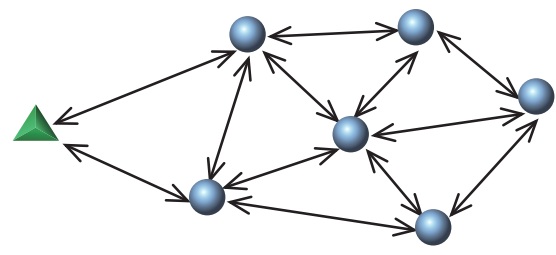

:End device (Measurement, Data transmission, relay)

:Coordinator (Data reception)

(a) General sensor network

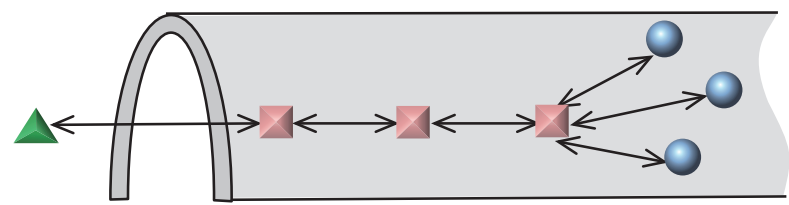

:End device (Measurement, Data transmission)

:Coordinator (Data reception)

:Relay wireless sensor

(b) Network with relay sensor

Fig. 7 Data transmission of wireless sensor

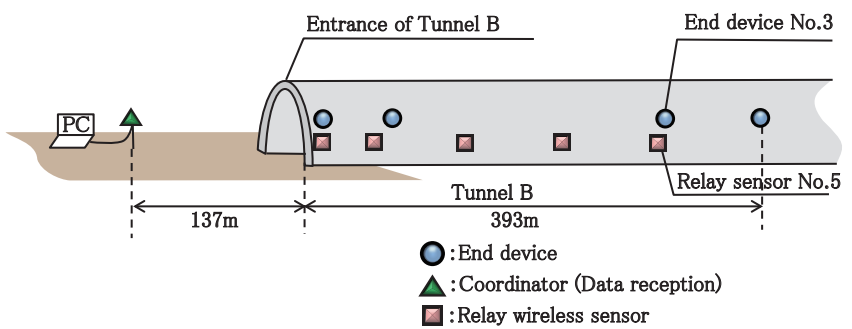

Fig. 8 Outline of the field test with relay sensors [5]

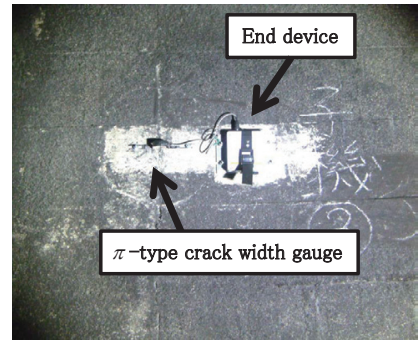

(a) End device

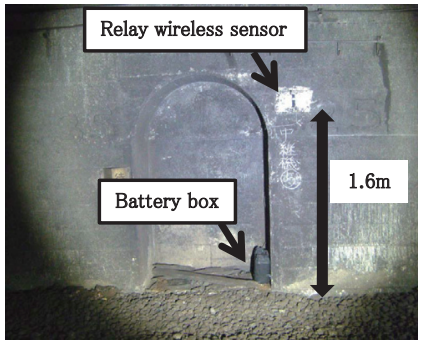

(b) Relay wireless sensor
Fig. 9 Installation situation of wireless sensors

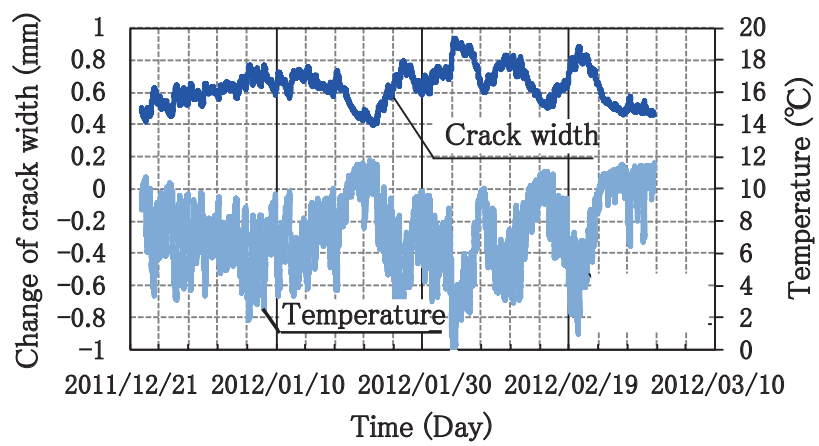

(a) End device No.3 (324m from the tunnel entrance)

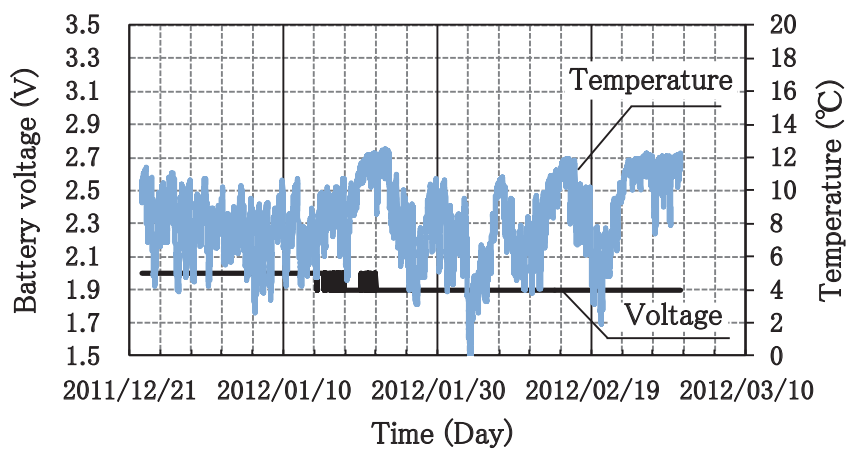

(b) Relay wireless sensor No.5 (305m from the tunnel entrance)

Fig. 10 Example of measurement data

on the tunnel lining as shown in Fig. 9 (a). The relay sensors are powered by 44 size $\mathrm{D}$ batteries.

Every 5 minutes, the measurement data for crack width, temperature, and battery voltage is transmitted from the end devices to the coordinator, and the data for temperature and battery voltage is likewise transmitted from the relay sensors to the coordinator. The data obtained by end device 3 and relay sensor 5 are shown in Fig. 10, which indicates that crack width becomes slightly large as temperature reduces. It is verified that it is possible to 
transmit the measurement data using the proposed system incorporating relay sensors.

\subsection{Investigation of power saving}

The firmware of wireless sensors used during the field test is improved in order to achieve further power-savings for sensors [4]. The electrical power control of the circuit for data acquisition, data transmission, analog-digital conversion and others is made more efficient to save power, while the frequency of transmission of the same data is reduced from three times to once by improving the irrefragability of data transmission just after the wake-up of the end devices. The measurement accuracy is also improved.

Figure 11 shows the battery voltage reduction for the end devices with new firmware and those with existing firmware obtained by a laboratory simulation test. The end devices are activated with two LR44-type button alkaline batteries and data are acquired every 5 minutes in order to rapidly consume the power of batteries. Test results in Fig. 11 show that the introduction of new firmware extends battery life dramatically. The capacity of LR44-type button alkaline batteries is $150 \mathrm{mAh}$. The level of consumed power for existing and new firmware is $0.022 \mathrm{~mW}$ and $0.005 \mathrm{~mW}$, respectively, for each unit of data (28Byte). As the transmission frequency of the same data is reduced from three

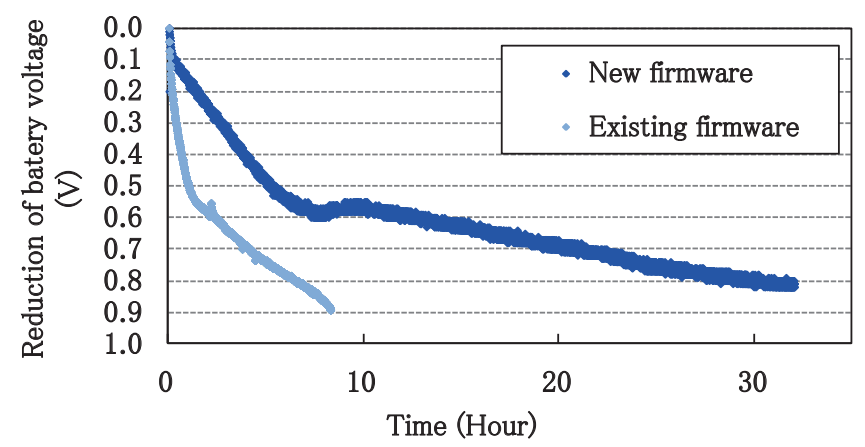

Fig. 11 Change of battery voltage

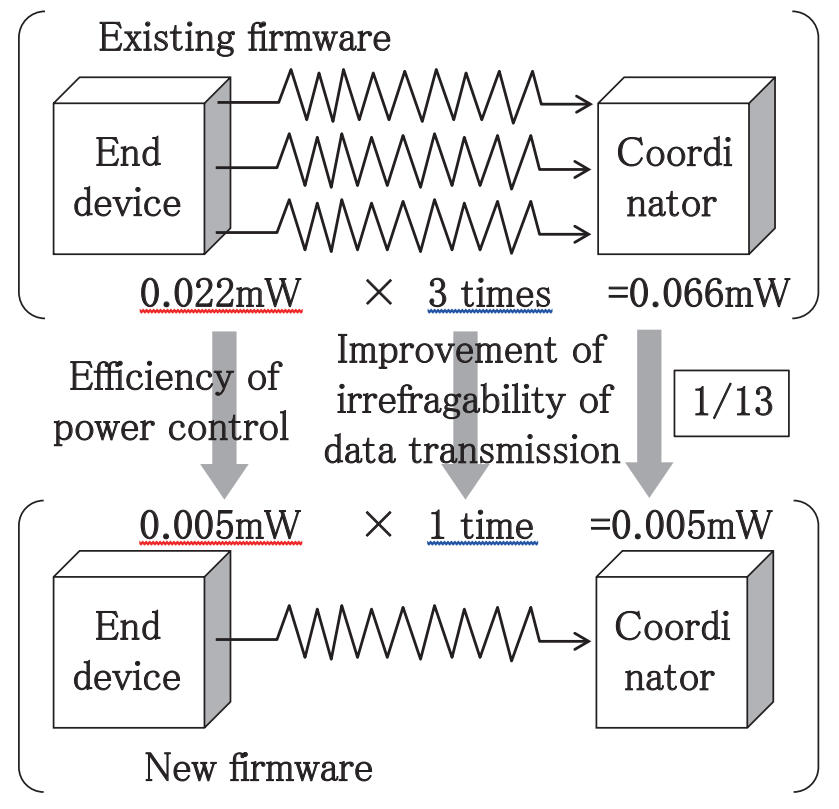

Fig. 12 Comparison of power consumption times to once, consumed power decreases by a thirteenth as shown in Fig. 12 by means of the introduction of new firmware. Considering the durable period of the components and the batteries, monitoring may finish before the batteries go flat. Nevertheless, it is found that there is a possibility of extending battery life dramatically by means of the development of firmware.

\section{Method for processing monitored data}

\subsection{Frequency analysis of measurement data}

The measurement data for crack with and temperature as shown in Fig. 5 are regarded as time-domain wave and analyzed by Fourier transform. Figure 13 shows the relationship between periods and Fourier coefficients regarding the measurement data for crack width for 180 days. The outline of utilized data is shown in Table 1. As the event probability of data defects is less than $0.1 \%$, the sampling intervals described in the table are calculated by dividing the data acquisition period by the number of

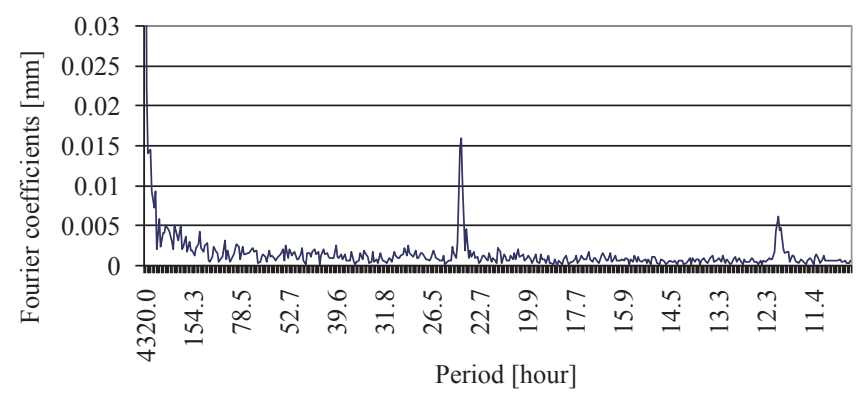

(a) No.1 (near the tunnel entrance)

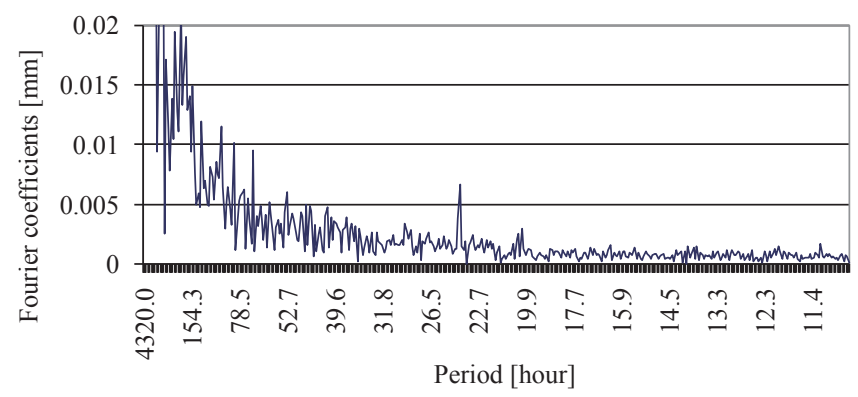

(b) No.2 (15m from the tunnel entrance)

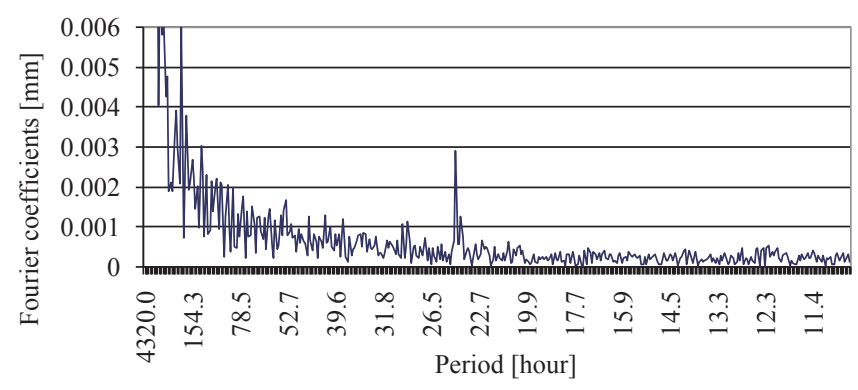

(c) No.3 (87m from the tunnel entrance)

Fig. 13 Relationship between periods and Fourier coefficients (crack width) 
Table 1 Outline of the analyzed data

\begin{tabular}{|c|c|c|c|c|}
\hline No. & $\begin{array}{c}\text { Distance from } \\
\text { tunnel entrance }\end{array}$ & $\begin{array}{c}\text { Data acquisition } \\
\text { period }\end{array}$ & Number of data & $\begin{array}{c}\text { Average } \\
\text { sampling interval }\end{array}$ \\
\hline 1 & Near tunnel entrance & 180 days & 30,695 & $8 \mathrm{~min} 27 \mathrm{sec}$ \\
\hline 2 & $15 \mathrm{~m}$ & 180 days & 30,786 & $8 \mathrm{~min} 25 \mathrm{sec}$ \\
\hline 3 & $87 \mathrm{~m}$ & 180 days & 30,206 & $8 \mathrm{~min} 35 \mathrm{sec}$ \\
\hline
\end{tabular}

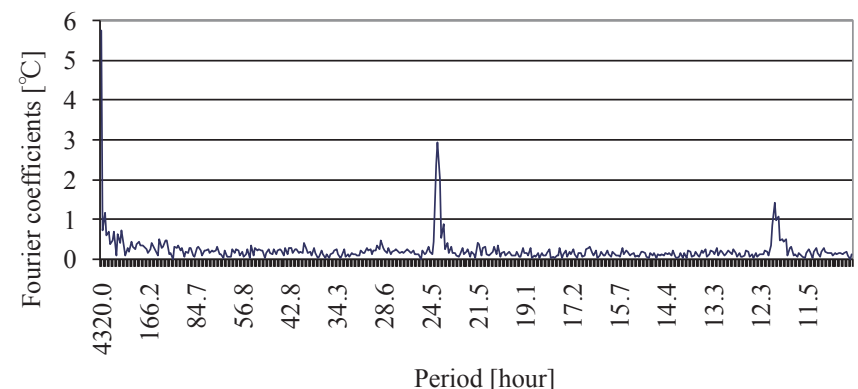

(a) No.1 (near the tunnel entrance)

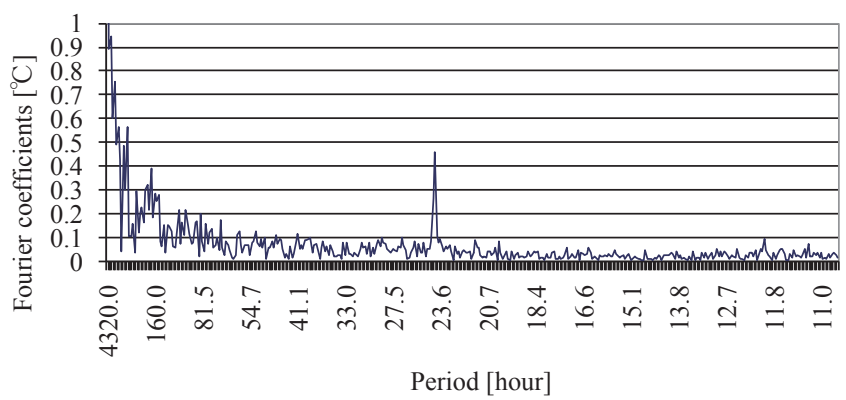

(b) No.2 (15m from the tunnel entrance)

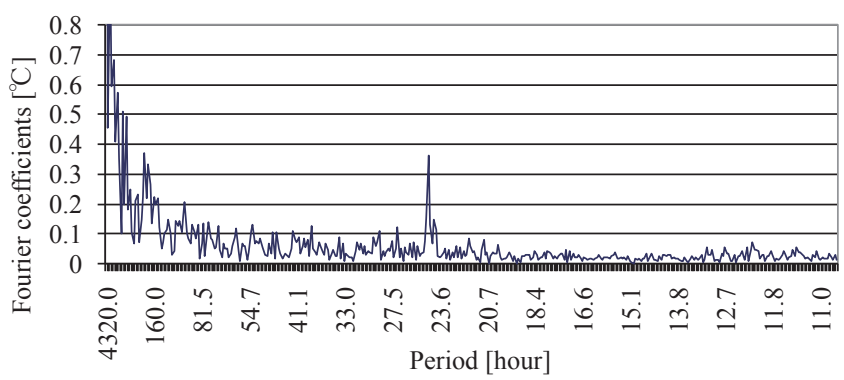

(c) No.3 (87m from the tunnel entrance)

Fig. 14 Relationship between periods and Fourier coefficients (temperature)

data. It is found that absolute peaks are observed just at 24 hours and this tendency becomes more pronounced near the tunnel entrance. The analyzed results for temperature shown in Fig. 14 indicate a similar tendency to those for crack width. It can be considered that the peaks observed in the crack width spectrum are related to daily temperature changes and become more pronounced near the tunnel entrance, where the data are affected by daily temperature changes.

\subsection{Data processing method based on frequency analysis}

Measurement data obtained by means of automatic monitoring include short-term variations caused by daily temperature changes. Short period components in the spectra obtained by means of the frequency analysis of measurement data do not affect long-term variations, which are considered to be quite important for the grasp of malfunction such as tunnel deformation. As clear peaks are observed just at 24 hours in the spectra, measurement data are processed with a low-pass filter which passes the components more than 24 hours in order to eliminate the short-period components. Measurement data are analyzed by means of Fourier transformation in the same way as described in section 3.1, processed with a low-pass filter of seven days and analyzed again with Fourier inverse transform to obtain a processed wave.
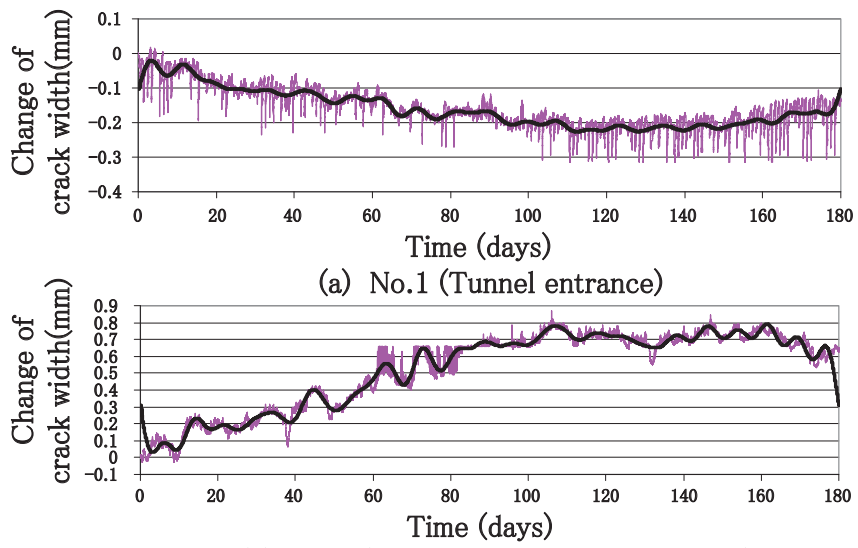

(b) No.2 (15m from the tunnel entrance)

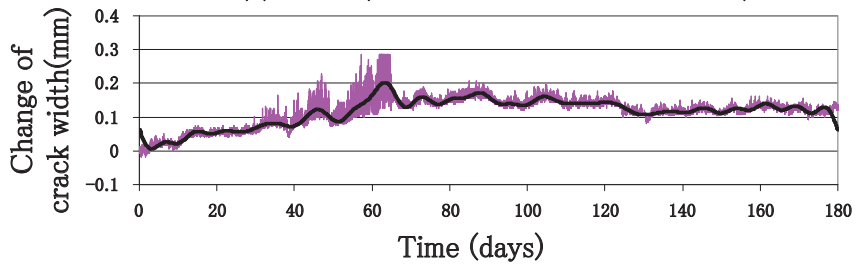

(c) No.3 (87m from the tunnel entrance)

Fig. 15 Results of data processing (crack width)

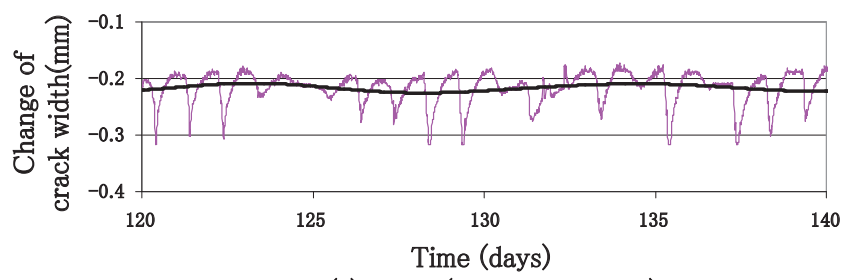

(a) No.1 (Tunnel entrance)

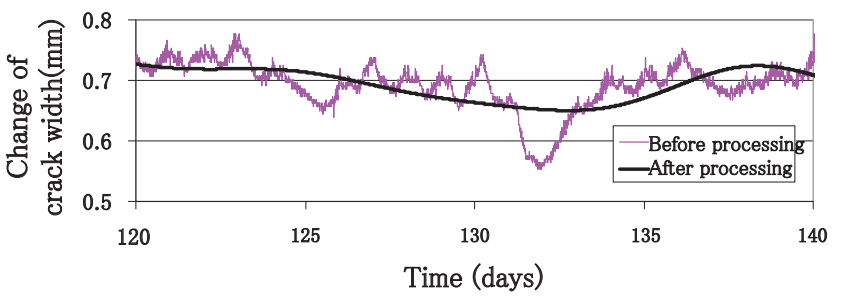

(b) No.2 (15m from the tunnel entrance)

Fig. 16 Results of data processing (crack width) 
The processed data are compared with raw data as shown in Fig. 15 and Fig. 16. Figure 16 shows the results for 20 days regarding the change in crack width at No.1 and 2. It is found that the short-term variations observed in the raw data are eliminated by the data processed using the low-pass filter. As mentioned above, it is verified that the short-term variations caused by daily temperature changes can be eliminated by processing measurement data using a low-pass filter.

\section{Conclusions}

This research investigated a tunnel monitoring method based on a wireless sensor network. The following results were obtained from the investigation.

1) A monitoring method using a wireless sensor network was developed to lighten the data acquisition workload and reduce the costs for monitoring railway tunnels. The applicability of the proposed method to actual sites was verified by means of a field test at a railway tunnel.

2) A data transmission method using relay wireless sensors was developed to transmit measurement data over a long distance. The applicability of the proposed method to actual sites was verified by means of a field test at a railway tunnel.

3) It was found that there is a possibility of saving battery power dramatically by means of the development of firmware.

4) Spectra obtained by analyzing the measurement data of crack width with Fourier transform has absolute peaks just at 24 hours, which is considered to be attributable to daily temperature changes.

5) It was verified that the short-term variations caused by daily temperature changes and others can be eliminated by processing measurement data using a low-pass filter.

\section{References}

[1] Tsuno, K., Kamachi, H., Nakanishi, Y., and Nakayama, T., "Monitoring system for deformed tunnel using wireless sensor network," Proceedings of Tunnel Engineering, JSCE, Vol. 19, pp. 245-249, 2009 (in Japanese).

[2] Tsuno, K. and Funahashi, T., "Application of Sensor Technology and Wireless Communication to Monitoring of Deformed Tunnels," The Journal of the Institute of Electrical Engineers of Japan, Vol. 131, No. 2, pp. 92-95, 2011 (in Japanese).

[3] Funahashi, T., Tsuno, K., Kamachi, H., Itoh, T., Fukushi, J., and Nakanishi, Y., "Application of tunnel monitoring system with wireless sensor network to actual railway tunnel," Proceedings of $65^{\text {th }}$ Annual Conference of the Japan Society of Civil Engineers, VI, pp. 87-88, 2010 (in Japanese).

[4] Tsuno, K., Hirata, R., Fukushi, J., Matsumoto, T., and Kamachi, H., "Investigation of power saving method of tunnel monitoring system," Proceedings of $67^{\text {th }}$ Annual Conference of the Japan Society of Civil Engineers, CS8, pp. 33-34, 2012 (in Japanese).

[5] Hirata, R., Tsuno, K., Sumi, Y., Mizuochi, K., and Kamachi, H., "Investigation of long-distance data transmission method with relay wireless sensor," Proceedings of $67^{\text {th }}$ Annual Conference of the Japan Society of Civil Engineers, CS8, pp. 35-36, 2012 (in Japanese).

[6] Tsuno, K., Kamachi. H., and Funahashi, T., "Investigation of processing method of real-time data obtained by tunnel-distortion monitoring," Proceedings of Tunnel Engineering, JSCE, Vol. 21, pp. 189-194, 2011.

\section{Authors}

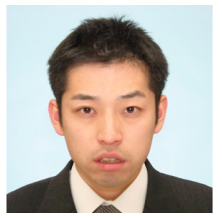

Kiwamu TSUNO, Dr. Eng.

Senior Researcher, Tunnel Engineering Laboratory, Structures Technology Division Research Areas: Maintenance and construction of urban tunnel, Subway-induced vibration

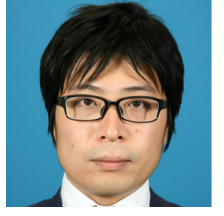

Ryo HIRATA

Researcher, Tunnel Engineering Laboratory, Structures Technology Division Research Areas: Maintenance and construction of mountain tunnel 